\title{
Identifying the Most Luminous QSOs in the Universe from Multiwavelength Sky Surveys
}

\author{
Amy Kimball, J. Condon, C. Lonsdale and M. Lacy \\ CSIRO Astronomy and Space Science, Australia \\ E-mail: Amy.Kimball@csiro.au
}

\begin{abstract}
I have combined data from sky surveys in the UV to the mid-IR, along with radio and X-ray data, to identify the most luminous QSOs in the Universe. The primary sky surveys were the Sloan Digital Sky Survey (SDSS) 7th Data Release QSO Catalog, which provides unambiguous broad-line QSO classification and robust redshifts, and the Wide-field Infrared Survey Explorer (WISE) mid-IR catalog, because a large percentage of QSO bolometric luminous emerges in the IR. Out of the 100,000 SDSS/WISE QSOs, we find $140(<0.2 \%)$ with bolometric luminosity greater than $2 \times 10^{14} \mathrm{Lo}$, with redshifts ranging from about 1.7 to 5 . The most luminous QSO found has Lbol $\approx 7 \times 10^{14}$ Lo. Merger-based galaxy evolution models predict that the host galaxies of such sources at peak QSO luminosity are undergoing a short-lived phase of extreme AGN feedback and massive star-formation activity after a major merger. Upcoming sub-mm observations with the new Atacama Large Millimeter/Sub-millimeter Array (ALMA), for a subset of the sample, will soon reveal crucial host galaxy properties of this unique sample.
\end{abstract}

